\section{Mount Everest Base Camp Medical Clinic "Everest ER": Epidemiology of Medical Events During the First 10 Years of Operation}

Objective.-As the highest peak on the planet, Mount Everest provides a truly austere environment in which to practice medicine. We examined records of all visits to the Everest Base Camp Medical Clinic (Everest ER) to characterize the medical problems that occur in these patients.

Methods.-A retrospective analysis of medical records from the first 10 years of operation (2003-2012) was performed. Descriptive data collected included patient demographics, diagnoses, treatments, prescriptions, medications dispensed, and evacuation type, if any.

Results.-In all, 2941 patients were seen for a total of 3569 diagnoses. The number of patient visits each year at the Everest ER increased at a greater rate than the total numbers of climbers attempting Mount Everest over this period. The most commonly treated patients were Nepalese, accounting for $51 \%$ of all nationalities. The most common medical diagnoses were from pulmonary causes such as high altitude cough and upper respiratory infections, comprising more than $38 \%$ of all medical diagnoses. The most common traumatic diagnoses were from dermatologic causes such as frostbite and lacerations, comprising $56 \%$ of all traumatic diagnoses. Pulmonary and dermatologic diagnoses were also the most frequent reasons for evacuation from Everest Base Camp, most commonly for high altitude pulmonary edema and frostbite, respectively.

Conclusions.-Medical professionals treating patients at extreme altitude should have a broad scope of practice and should be well prepared to deal with serious traumas from falls, cold exposure injuries, and altitude illness.

Andrew B. Pressman, MD Royal Oak, MI, USA Mária Némethy, MD Scott E. McIntosh, MD, MPH Salt Lake City, UT, USA Luanne Freer, MD Everest, Nepal, and Yellowstone National Park, WY, USA

\section{Development and Implementation of a Standar- dized Wilderness Medicine Curriculum (AWLS) for Fourth Year Medical Students}

Introduction.-A wilderness medicine course was implemented in a 2-week period of a fourth-year medical student rotation at the Medical University of South Carolina in 2012. Previously, there had been no offered course for medical students of any year of training.

Objective.-The purpose of this review is to describe the development and implementation of this curriculum so that other programs may use it as a template for their own institutional experience.

Methods.-This report is a description of the development and implementation of our wilderness medicine course. We used a standardized, preexisting curriculum with the addition of supplementary pathophysiology that one could encounter in the field of wilderness medicine. Emergency medicine (EM) resident physicians and pediatric EM fellows were involved; the involvement of our hospital legal team and the collection of additional fees are also reviewed.

Results. - The process of implementation and adaptation of this preexisting wilderness medicine course for a specific audience of fourth-year medical students is described. The potential for this curriculum to be incorporated into a more comprehensive longitudinal learning experience during the students' subsequent residency training was also taken into consideration. Examples of didactic teaching and hands-on assessment modalities are discussed in detail, as well as results from testing and follow-up student surveys. The course was well regarded by the students.

Conclusions.-A wilderness medicine curriculum was successfully established for fourth-year medical students at our institution. Students believe the course enhanced their medical school education, and many would continue to maintain their certification. A few intend to further their wilderness medicine education by becoming instructors. Areas of potential future growth of the course and development of a more comprehensive longitudinal curriculum were identified.

Simon C. Watson, MD

Sarah E. Sterner, MD

Jeffrey S. Bush, MD

Christina L. Bourne, MD

Charleston, SC, USA 